\title{
Magnetic resonance-guided laser interstitial thermal therapy for brain tumors in geriatric patients
}

\author{
Elizabeth E. Ginalis, MD, and Shabbar F. Danish, MD \\ Department of Neurological Surgery, Rutgers Robert Wood Johnson Medical School, New Brunswick, New Jersey
}

OBJECTIVE There is a paucity of studies assessing the use of MR-guided laser interstitial thermal therapy (LITT), specifically in the elderly population. The aim of this study was to evaluate the safety of LITT for brain tumors in geriatric patients.

METHODS Geriatric patients ( $\geq 65$ years of age) treated with LITT for intracranial tumors at a single institution between January 2011 and November 2019 were retrospectively identified. The authors grouped patients into two distinct age cohorts: 65-74 years (group 1) and 75 years or older (group 2). Baseline characteristics, operative parameters, postoperative course, and morbidity were recorded for each patient.

RESULTS Fifty-five geriatric patients underwent 64 distinct LITT procedures for brain tumors. The majority of lesions $(40[62.5 \%])$ treated were recurrent brain metastases or radiation necrosis. The median modified frailty index was 0.1 (low frailty; range $0-0.4$ ) for patients in group 1 and 0.2 (intermediate frailty; range $0-0.4$ ) for patients in group 2 ( $p>$ 0.05 ). The median hospital length of stay (LOS) was 1 day (IQR 1-2 days); there was no significant difference in LOS between the age groups. The hospital stay was significantly longer in patients who presented with a neurological symptom and in those who experienced a postoperative complication. The majority of patients (43 [68.3\%] of 63 cases) were fit for discharge to their preoperative accommodation following LITT. The rate of discharge to home was not significantly different between the age groups. Those discharged to rehabilitation facilities were more likely to have presented with a neurological symptom. Nine patients ( $14.1 \%$ of cases) were found to have acute neurological complications following LITT, with nearly all patients showing complete or partial recovery at follow-up. The 30-day postoperative mortality rate was $1.6 \%$ (1 case). The complication and 30-day postoperative mortality rates were not significantly different between the two age groups.

CONCLUSIONS LITT can be considered a minimally invasive and safe neurosurgical procedure for the treatment of intracranial tumors in geriatric patients. Careful preoperative preparation and postoperative care is essential as LITT is not without risk. Appropriate patient selection for cranial surgery is essential, because neurosurgeons are treating an increasing number of elderly patients, but advanced age alone should not exclude patients from LITT without considering frailty and comorbidities.

https://thejns.org/doi/abs/10.3171/2020.7.FOCUS20462

KEYWORDS brain tumors; geriatrics; laser ablation; laser interstitial thermal therapy

$\mathrm{G}$ LOBAL population aging has led to an increase in the geriatric population worldwide. This is in part due to the decrease in mortality at older ages and the consequent increase in life expectancy. In the United States, the number of geriatric people-aged 65 years and older-is expected to nearly double from 52 million in 2018 to 95 million by 2060; specifically, the number of people aged 85 and older will triple during that same time period. ${ }^{1}$ At this rate, the elderly population will compose approximately $23 \%$ of the total population in the United States. ${ }^{1}$
This expanding geriatric population has led to an increase in surgical procedures in the elderly., ${ }^{2,3}$ However, older age has been found to be a risk factor for surgeryrelated adverse events, worse outcomes, and postoperative mortality. ${ }^{4-7}$ Additionally, these patients often present with a greater number of comorbidities, again resulting in a higher risk of postoperative complications. ${ }^{3,4}$ Consequently, minimally invasive approaches, such as MR-guided laser interstitial thermal therapy (LITT), are attractive treatment options for geriatric patients, because compared

ABBREVIATIONS CCI = Charlson Comorbidity Index; COPD = chronic obstructive pulmonary disease; GBM = glioblastoma multiforme; IQR = interquartile range; LITT = laser interstitial thermal therapy; LOS = length of stay; $\mathrm{mFI}=$ modified frailty index.

SUBMITTED May 29, 2020. ACCEPTED July 20, 2020.

INCLUDE WHEN CITING DOI: 10.3171/2020.7.FOCUS20462. 
to open surgery they result in shorter hospital stays, increased rates of discharge to home, fewer complications, and decreased cost of hospitalization. ${ }^{8-10}$ Moreover, LITT can be considered in those elderly patients who are not surgical candidates, have exhausted alternative treatment methods, or present with difficult-to-access lesions in surgically challenging locations.

Understanding the impact of cranial surgery in the geriatric patient can lead to improved surgical outcomes as the population continues to age. Various reports have described clinical outcomes in elderly patients following neurosurgical procedures., ${ }^{4,5,711,12}$ However, there is currently a paucity of data regarding the use of LITT in geriatric patients specifically. In this study, we evaluated the safety and clinical outcomes of geriatric patients undergoing LITT for brain tumors. To our knowledge, this is the first study in which short-term outcomes and mortality rates in the geriatric neurosurgical patient following LITT were analyzed.

\section{Methods}

Geriatric patients ( $\geq 65$ years of age) who had been treated with MR-guided LITT for intracranial tumors, using the Visualase thermal therapy system (Medtronic) ${ }^{13}$ at a single institution (Rutgers Robert Wood Johnson University Hospital) between January 2011 and November 2019 , were retrospectively identified and included in the study. Institutional review board approval for the study was obtained. All procedures had been performed by the senior author, and our operative procedure was previously described..$^{14,15}$ In 11 patients, a needle biopsy was obtained at the time of ablation. Postoperative clinical monitoring was performed in the ICU, in the step-down unit, or on the neurosurgical floor.

Patients were offered LITT for their diagnoses in the following instances: 1) the diagnosis was not controlled with other therapies (including open resection and/or radiation treatment); 2) the tumor was considered inoperable (could not be safely resected); and/or 3) the patient preferred LITT over other treatments. Generally, LITT was offered as part of a clinical care algorithm determined by a multidisciplinary board.

The definition of "elderly" is inconsistent across studies. ${ }^{4,5,12}$ We defined elderly patients as those who were 65 years of age or older. We grouped patients into two discrete age cohorts: 65-74 years (group 1) and 75 years or older (group 2). In this way, we defined an advanced elderly group and compared the two age cohorts.

Baseline characteristics, operative parameters, postoperative course, discharge destination, and mortality were recorded for each patient. Calculation of frailty and comorbidity indices for each patient was conducted to assess preoperative risk. To this aim, we used the modified frailty index (mFI) described in the study by Youngerman et al. ${ }^{16}$ and the Charlson Comorbidity Index (CCI), which predicts 10-year survival in patients with multiple comorbidities. Eleven variables (morbid conditions) are assessed in determining the mFI. One point is assigned to each condition found to be present in the patient; the total number of conditions is then divided by the total number of variables recorded for that patient..$^{5,16-18}$ The $\mathrm{mFI}$ ranged from 0 to 1 , and was organized into 4 categories: no frailty (0), low frailty $(0.01-0.19)$, intermediate frailty $(0.2-0.29)$, and high frailty $(\geq 0.3)$. Any variables for which we did not have patient data were excluded from the mFI calculation.

For patient and treatment characteristics, summary statistics were calculated via frequencies and percentages for categorial data and either means ( \pm standard deviations) or medians (interquartile ranges [IQRs] or ranges) for continuous variables. Characteristics were compared using a t-test for continuous variables and the Fisher test or chisquare test for categorical variables. A p value $<0.05$ was considered statistically significant. All data analysis was performed using Microsoft Excel version 16.33.

\section{Results}

\section{Patient Characteristics}

In total, 55 patients underwent 64 distinct LITT procedures. Thirty-six women (56.2\% of cases) were included in the study. The median patient age was 70 years (range 65-90 years; average $72.7 \pm 6.3$ years). There were 47 cases in age group 1 (65-74 years) and 17 cases in age group 2 ( $\geq 75$ years). The majority $(53.3 \%)$ of cases presented with neurological symptoms. The median $\mathrm{mFI}$ was 0.1 (low frailty: range 0-0.4) in group 1 and 0.2 (intermediate frailty: range $0-0.4$ ) in group 2 . This difference was not statistically significant. The median CCI was 8 (mean 7.3 \pm 2.4 ) in group 1 and 9 (mean $8.8 \pm 2.7$ ) in group 2, both corresponding to a $0 \% 10$-year survival rate. This difference in CCI was statistically significant ( $\mathrm{p}<0.05)$. In total, $19 \%$ of cases had a baseline significant comorbidity (chronic obstructive pulmonary disease [COPD], congestive heart failure, previous myocardial infarction, or previous stroke). The most common comorbidity was hypertension (65.1\%), followed by diabetes $(17.5 \%)$ and COPD $(15.9 \%)$. Many cases included current or former smokers $(46 \%)$. In the majority of cases $(62.9 \%)$, the patients were overweight, with a median body mass index of $26.8 \mathrm{~kg} / \mathrm{m}^{2}$. Table 1 summarizes patient demographics, clinical characteristics, and outcomes.

The majority of lesions (40 [62.5\%]) that were treated were recurrent brain metastases or radiation necrosis. We generally do not obtain a biopsy as part of these procedures. Particularly for prior treated metastatic tumors, we classify them together as in-field recurrences or PIERS (progressive inflammatory enhancement after radiosurgery). ${ }^{19}$ Glioblastoma multiforme (GBM) was the next most common pathological lesion treated with LITT (19 cases [29.7\%]). Other primary diagnoses included meningioma, oligodendroglioma, CNS lymphoma, ependymoma, and hemangioblastoma (1 case [1.6\%] each). Table 2 shows the rates of mortality, complications, and discharge to home stratified by primary diagnosis. Sites of ablation included the frontal lobe (34.4\%), parietal lobe (25\%), temporal lobe $(17.2 \%)$, cerebellum $(15.6 \%)$, occipital lobe $(3.1 \%)$, brainstem $(3.1 \%)$, and corpus callosum $(1.6 \%)$. In the majority of cases $(88.1 \%)$, the patients had received some intervention for their tumor before undergoing LITT. 
TABLE 1. Patient demographics, clinical characteristics, and outcomes

\begin{tabular}{|c|c|c|c|}
\hline Characteristic & All Ages & Group 1 (65-74 yrs) & Group 2 ( $\geq 75$ yrs) \\
\hline No. of cases & $64(100)$ & $47(73.4)$ & $17(26.6)$ \\
\hline Age, yrs (range) & $70(65-90)$ & $69(65-74)$ & $80(75-90)$ \\
\hline Female & $36(56.3)$ & $25(53.2)$ & $11(64.7)$ \\
\hline \multicolumn{4}{|l|}{ Primary diagnosis } \\
\hline Primary intracranial tumor & $24(37.5)$ & $19(40.4)$ & $5(29.4)$ \\
\hline Metastasis or radiation necrosis & $40(62.5)$ & $28(59.6)$ & $12(70.6)$ \\
\hline Baseline neurological symptoms present* & $32(53.3)$ & $27(60)$ & $5(33.3)$ \\
\hline $\mathrm{mFI}$ & $0.11(0.1-0.2)$ & $0.1(0.1-0.2)$ & $0.2(0.075-0.205)$ \\
\hline $\mathrm{CCl}$ & $8(5-8)$ & $8(4-9)$ & $9(6-10)$ \\
\hline 30-day mortality & $1(1.6)$ & $1(2.1)$ & $0(0)$ \\
\hline Overall mortality & $5(7.8)$ & $4(8.5)$ & $1(5.9)$ \\
\hline ICU LOS, days & $0(0-1)$ & $0(0-1)$ & $0(0-0)$ \\
\hline Hospital LOS, days & $1(1-2)$ & $1(1-2)$ & $1(1-1)$ \\
\hline \multicolumn{4}{|l|}{ Discharge destination $†$} \\
\hline Home or preop accommodation & $43(68.3)$ & $32(68.1)$ & $11(68.8)$ \\
\hline Rehabilitation facility & $20(31.7)$ & $15(31.9)$ & $5(31.2)$ \\
\hline
\end{tabular}

Values are presented as the number of cases (\%) or as the median (IQR) unless indicated otherwise.

* Data available for 60 cases, 45 in group 1 and 15 in group 2.

† Data available for 63 cases, 47 in group 1 and 16 in group 2.

\section{Surgical and Postoperative Care}

The median ablation time was 2.75 minutes (range 0.87-30.9 minutes; average $8.6 \pm 5.7$ minutes), and the median ablation power was $11.25 \mathrm{~W}$ (range 6-20 W; average $11.4 \pm 1.9 \mathrm{~W}$ ). The estimated blood loss was minimal $(<50 \mathrm{ml})$ in all cases. The median hospital length of stay (LOS) was 1 day (IQR 1-2 days; average $1.75 \pm 1.5$ days). Statistical comparison of the two age groups revealed no significant difference in hospital LOS. The presence of a neurological symptom at baseline and the presence of a postoperative complication were each associated with significantly longer hospital stays $(\mathrm{p}<0.05)$. The majority of patients were not monitored in the ICU postoperatively, and advanced age did not mandate an ICU admission.

There was 1 case of reoperation within 48 hours after LITT. The patient, a 65-year-old man treated for hemangioblastoma, was among the earlier patients treated with LITT at our facility. In his case, LITT was aborted due to inaccurate laser placement. Consequently, the patient underwent a suboccipital craniotomy for tumor resection.

In the majority of cases $(43 / 63[68.3 \%])$, the patients were fit for discharge to home or their preoperative ac- commodation (i.e., assisted living facility) following LITT. In the remaining cases (20/63 [31.7\%]), the patients were discharged to a rehabilitation facility. There was no statistically significant difference in discharge destination between the two age groups. There was also no significant difference in $\mathrm{mFI}$ or CCI between those patients who were discharged home and those who were discharged to a rehabilitation facility. Patients with metastatic tumors were significantly more likely to be discharged home (79.5\% of cases) than those with primary intracranial tumors (54.2\% of cases). Patients who were discharged to rehabilitation facilities were significantly more likely to have presented with a neurological symptom ( $83.3 \%$ of cases) than those who were discharged to home ( $40.5 \%$ of cases).

\section{Adverse Effects and Mortality}

There was one perioperative complication due to inaccurate laser placement, which was described previously. Nine patients (14.1\% of cases) were found to have acute neurological complications following LITT; these included increased weakness $(n=7)$, aphasia $(n=2)$, confusion due to symptomatic cerebral edema $(\mathrm{n}=1)$, and cognitive

TABLE 2. Thirty-day mortality, overall mortality, complications, and discharge destination stratified by primary diagnosis

\begin{tabular}{llccc}
\hline \multicolumn{1}{c}{ Pathology } & $\begin{array}{c}30 \text {-Day } \\
\text { Mortality }\end{array}$ & $\begin{array}{c}\text { Overall } \\
\text { Mortality }\end{array}$ & Complications & $\begin{array}{c}\text { Discharge to Home or } \\
\text { Preop Accommodation }\end{array}$ \\
\hline GBM & $0(0)$ & $1(5.3)$ & $1(5.3)$ & $10(52.6)$ \\
\hline Other primary brain tumor & $0(0)$ & $0(0)$ & $2(40)$ & $3(60)$ \\
\hline Intracranial metastasis or radiation necrosis & $1(2.5)$ & $4(10)$ & $7(17.9)$ & $30(76.9)$ \\
\hline
\end{tabular}

Values are presented as number (\%). 
deficits $(\mathrm{n}=1)$. In 3 cases $(33.3 \%)$ the patients experienced complete resolution of the neurological deficit, in 5 cases (55.6\%) they experienced partial resolution, and in the last case (11.1\%) the patient died without resolution of the deficit. The complication rate was not statistically significant between the two age groups. The $\mathrm{mFI}, \mathrm{CCI}$, presence of baseline neurological symptoms, pathology type, and ablation time were not significantly different between those patients who experienced complications and those who did not have complications.

At the time of this study, 5 patients had died, corresponding to an overall mortality rate of $7.8 \%$ of cases. The cause of death was neurological in 2 cases (3.1\%). Four of these patients were treated for brain metastasis and 1 patient for GBM. The median age at the time of death was 70 years (range 66-75 years). These patients died a median of 1.9 months (range 0.36-12.9 months) following their first LITT. The 30-day postoperative mortality rate was $1.6 \%$. Table 3 summarizes the characteristics of the deceased patients.

\section{Discussion}

As the population continues to age, we must understand the impact of neurosurgical intervention on geriatric patients so that their cases can be managed safely and appropriately. The purpose of this study was to evaluate the safety of LITT in geriatric patients to treat brain tumors. Our patients' complication and mortality rates are comparable to those of other LITT studies. Most of our patients were discharged home postoperatively after a short hospital LOS with comparable morbidity rates. LITT appears to be a safe, minimally invasive alternative for geriatric patients with intracranial tumors.

Various studies have described outcomes in elderly patients following neurosurgical procedures, although the age for inclusion varies across those studies, ${ }^{4,5,7,11,12,16}$ Schär et al. reported an overall 30-day mortality rate of $0.68 \%$ in elderly patients ( $\geq 65$ years of age) undergoing elective cranial surgery. ${ }^{4}$ Bligh et al. found a 30 -day mortality rate of $0.8 \%$ in elderly patients ( $\geq 70$ years of age) following any elective neurosurgical procedure. ${ }^{12}$ However, the 30 day mortality rate among patients who underwent craniotomy for brain lesion excision was $8.1 \%$, suggesting that mortality rates vary depending on the type of surgery that was performed. In our study, there was only one death within 30 days following LITT, resulting in a 30-day mortality rate of $1.6 \%$. This death was not directly related to
LITT. Although Hong et al. did not exclusively study geriatric patients, they found no difference in overall survival between patients treated with LITT and those treated with craniotomy. ${ }^{20}$ Similarly, the mortality rate that we found with LITT in geriatric patients is comparable to that in the literature on craniotomy in geriatric patients. Additionally, it is similar to mortality rates in other LITT studies..$^{14,21,22}$ Given the frequent coexistence of chronic diseases among our patient cohort and the poor prognosis of many of their primary diseases, we found this to be an acceptable rate with regard to cranial surgery in the geriatric patient.

Interestingly, both Schär et al. and Bligh et al. found no statistically significant difference in 30-day mortality rates between their "advanced" elderly group and "younger" elderly group.,12 Similarly, in our study, older age was not associated with an increased mortality rate after LITT (age group 1 compared to age group 2). There was only 1 patient who died within 30 days of craniotomy, and he was younger than 75 years. The overall mortality rate in our study was $7.8 \%$ of cases, and only 1 of the 5 deaths occurred in the advanced elderly group, suggesting advanced age should not exclude patients from LITT.

There were 9 patients ( $14.1 \%$ of cases) who experienced an acute neurological complication following LITT, but nearly all of them showed complete or partial recovery at follow-up. This rate is similar to other studies in which complication rates have been reported following LITT, ranging from $6 \%$ to $26.7 \% .^{8,9,13-15,21-25}$ While we believe that the overall risks of LITT in the geriatric patient, with respect to both acute complications and 30-day mortality rate, are acceptable to recommend intervention, these risks must be weighed against the benefits of LITT. This risk-to-benefit ratio must be individually assessed for each patient. Further research on the topic of LITT efficacy in the geriatric population is needed.

The median LOS was shorter than that reported after craniotomy, which averages about 5 days.,26 Moreover, in a recent study, Schär et al. reported a median hospital LOS of 7 days in their geriatric patients who were 75 years of age and older, which was significantly longer than the LOS in their nongeriatric patients $\left(<65\right.$ years of age) ${ }^{4}$ Even though we found that the hospital stay was significantly longer in patients who presented with a neurological symptom (median 1 day) and in those who experienced a postoperative complication (median 3 days), the stay was still shorter than the median LOS reported for craniotomy. Minimally invasive neurosurgical interventions such as LITT are especially attractive in the geriatric patient, as

TABLE 3. Characteristics of the deceased patients

\begin{tabular}{ccrrrccc}
\hline $\begin{array}{c}\text { Age } \\
\text { (yrs) }\end{array}$ & Sex & $\mathrm{mFl}$ & $\mathrm{CCl}$ & Primary Diagnosis & $\begin{array}{c}\text { Time of Death } \\
\text { After LITT (days) }\end{array}$ & Cause of Death \\
\hline 66 & $\mathrm{~F}$ & 0.1 & 4 & GBM & 176 & Progression of intracranial disease \\
\hline 69 & $\mathrm{M}$ & 0.3 & 10 & Metastatic lung cancer (adenocarcinoma) & 25 & Cardiopulmonary arrest \\
\hline 70 & $\mathrm{~F}$ & 0.1 & 9 & Metastatic ovarian cancer & 57 & Progression of intracranial disease \\
\hline 70 & $\mathrm{~F}$ & 0.2 & 8 & Metastatic lung cancer (NSCLC) & 96 & Respiratory failure \\
\hline 75 & $\mathrm{M}$ & 0.2 & 10 & Metastatic lung cancer (NSCLC) & 45 & Respiratory failure \\
\hline
\end{tabular}

NSCLC $=$ non-small cell lung cancer. 
these approaches generally have a shorter LOS compared to traditional craniotomy.

Similarly, while we want to safely discharge patients to minimize hospital LOS, we simultaneously aim to safely discharge them to home when feasible. Discharging patients to their initial living arrangements is a measure of successful rehabilitation and is often perceived as an indicator of the quality of care. ${ }^{27}$ The majority of our patients were considered fit enough to return home or to their previous accommodation at discharge, and there was no significant difference between the age groups. However, Schär et al. found that the discharge rate to home was significantly lower in their advanced elderly ( $\geq 75$ years of age) group (44\%) compared to younger patients $(79 \%)$ following elective craniotomy. ${ }^{4}$ The rate of discharge for patients in the advanced elderly group in our study $(68.8 \%)$ is higher than that in the study conducted by Schär et al. (44\%). Additionally, in our study, patients discharged to rehabilitation facilities were more likely to have presented with a neurological symptom. Thus, the invasiveness of the approach (LITT compared with craniotomy) and the presence of neurological symptoms at baseline are two considerations that may impact the postdischarge destination.

Regarding health economics, LITT is a minimally invasive approach that could have favorable financial implications; this is particularly relevant because the geriatric population is responsible for a great proportion of healthcare costs and resources. One study revealed that LITT was attended by significantly less cost than craniotomy for metastatic brain tumors. ${ }^{28}$ However, there was no significant difference in cost for primary brain tumors between the two therapies. In another recent study, index hospitalization costs of LITT were significantly lower than those for open neurosurgical procedures for refractory epilepsy. ${ }^{10}$ Although beyond the scope of this paper, this suggests that LITT may be equal or possibly favorable to open surgery from an economic perspective. Additionally, we found that LITT was associated with shorter hospital and ICU stays as well as a greater rate of discharge to home compared to open craniotomy; these findings could theoretically contribute to the observed reduced cost of surgical care and subsequent economic burden on geriatric patients. However, it should be noted that in many instances, especially in neuro-oncology, LITT is not an alternative to craniotomy. For many of these patients, craniotomy is not an option due to the inaccessibility of the target. Consequently, a fair economic comparison may not be one that compares LITT to craniotomy, but one in which LITT is compared to the best medical care and one that takes into account days at home after surgery as a metric. ${ }^{29}$

\section{Study Limitations}

Our study has several limitations. First, it is a retrospective study involving patients from a single institution. Second, our small sample size limits statistical significance, and, consequently, we cannot make any definitive conclusions. Third, we did not define a control group (such as comprising ages $<65$ years) to make comparisons with our patient cohort. Similarly, we did not compare LITT to traditional neurosurgical interventions (such as craniotomy for tumor resection) for patients' diagnoses. Generally, open neurosurgical intervention or radiation therapy is the preferred treatment for brain tumors at our institution. Therefore, we believe that it is not necessarily appropriate to compare craniotomy to LITT, as LITT is generally a second-line treatment option that is offered to patients after traditional methods have failed to control the disease. Part of the inclusion criteria for LITT at our institution requires a high-performance status, which may introduce selection bias when evaluating this patient population.

\section{Conclusions}

LITT can be considered a minimally invasive and safe neurosurgical procedure for the treatment of intracranial tumors, even for geriatric patients. Careful preoperative preparation and postoperative care is essential because LITT is not without risk. Appropriate patient selection for cranial surgery is essential as neurosurgeons treat an increasing number of elderly patients. However, advanced age should not in itself exclude geriatric patients from undergoing LITT, if it is clinically indicated, without consideration of frailty, comorbidities, and functional status.

\section{References}

1. Mather M, Jacobsen LA, Pollard KM. Aging in the United States. Popul Bull. 2015;70(2):1-20.

2. Chibbaro S, Di Rocco F, Makiese O, et al. Neurosurgery and elderly: analysis through the years. Neurosurg Rev. 2010; 34(2):229-234.

3. Jablonski SG, Urman RD. The growing challenge of the older surgical population. Anesthesiol Clin. 2019;37(3):401-409.

4. Schär RT, Tashi S, Branca M, et al. How safe are elective craniotomies in elderly patients in neurosurgery today? A prospective cohort study of 1452 consecutive cases. J Neurosurg. Published online April 24, 2020. doi: 10.3171/2020.2.JNS193460

5. Tomlinson SB, Piper K, Kimmell KT, Vates GE. Preoperative frailty score for 30-day morbidity and mortality after cranial neurosurgery. World Neurosurg. 2017;107:959-965.

6. Patil CG, Veeravagu A, Lad SP, Boakye M. Craniotomy for resection of meningioma in the elderly: a multicentre, prospective analysis from the National Surgical Quality Improvement Program. J Neurol Neurosurg Psychiatry. 2010; 81(5):502-505.

7. Steinberger J, Bronheim RS, Vempati P, et al. Morbidity and mortality of meningioma resection increases in octogenarians. World Neurosurg. 2018;109:e16-e23.

8. Arocho-Quinones EV, Lew SM, Handler MH, et al. Magnetic resonance-guided stereotactic laser ablation therapy for the treatment of pediatric brain tumors: a multiinstitutional retrospective study. J Neurosurg Pediatr. 2020;26(1):13-21.

9. Sharma M, Ugiliweneza B, Wang D, et al. National trends and factors predicting outcomes following laser interstitial thermal therapy (LITT) for brain lesions: a Nationwide Inpatient Sample analysis. World Neurosurg. 2020;139:e88-e97.

10. Sharma M, Ball T, Alhourani A, et al. Inverse national trends of laser interstitial thermal therapy and open surgical procedures for refractory epilepsy: a Nationwide Inpatient Sample-based propensity score matching analysis. Neurosurg Focus. 2020;48(4):E11.

11. Stovell MG, Jenkinson MD. Neurosurgery in octogenarians. Br J Neurosurg. 2014;28(5):611-615.

12. Bligh ER, Sinha P, Smith D, Al-Tamimi YZ. Thirty-day mortality and survival in elderly patients undergoing neurosurgery. World Neurosurg. 2020;133:e646-e652.

13. Medvid R, Ruiz A, Komotar RJ, et al. Current applications 
of MRI-guided laser interstitial thermal therapy in the treatment of brain neoplasms and epilepsy: a radiologic and neurosurgical overview. AJNR Am J Neuroradiol. 2015;36(11): 1998-2006.

14. Patel P, Patel NV, Danish SF. Intracranial MR-guided laserinduced thermal therapy: single-center experience with the Visualase thermal therapy system. J Neurosurg. 2016;125(4): 853-860.

15. Jethwa PR, Barrese JC, Gowda A, et al. Magnetic resonance thermometry-guided laser-induced thermal therapy for intracranial neoplasms: initial experience. Neurosurgery. 2012; 71(1)(Suppl Operative):133-144, 144-145.

16. Youngerman BE, Neugut AI, Yang J, et al. The modified frailty index and 30-day adverse events in oncologic neurosurgery. J Neurooncol. 2018;136(1):197-206.

17. Lieber BA, Appelboom G, Taylor BE, et al. Preoperative chemotherapy and corticosteroids: independent predictors of cranial surgical-site infections. J Neurosurg. 2016;125(1): 187-195.

18. Parikh P, Shiloach M, Cohen ME, et al. Pancreatectomy risk calculator: an ACS-NSQIP resource. HPB (Oxford). 2010; 12(7):488-497.

19. Hernandez RN, Carminucci A, Patel P, et al. Magnetic resonance-guided laser-induced thermal therapy for the treatment of progressive enhancing inflammatory reactions following stereotactic radiosurgery, or PEIRs, for metastatic brain disease. Neurosurgery. 2019;85(1):84-90.

20. Hong CS, Deng D, Vera A, Chiang VL. Laser-interstitial thermal therapy compared to craniotomy for treatment of radiation necrosis or recurrent tumor in brain metastases failing radiosurgery. J Neurooncol. 2019;142(2):309-317.

21. Kamath AA, Friedman DD, Hacker CD, et al. MRI-guided interstitial laser ablation for intracranial lesions: a large single-institution experience of 133 cases. Stereotact Funct Neurosurg. 2017;95(6):417-428.

22. Kamath AA, Friedman DD, Akbari SHA, et al. Glioblastoma treated with magnetic resonance imaging-guided laser interstitial thermal therapy: safety, efficacy, and outcomes. Neurosurgery. 2019;84(4):836-843.

23. Gamboa NT, Karsy M, Iyer RR, et al. Stereotactic laser interstitial thermal therapy for brainstem cavernous malformations: two preliminary cases. Acta Neurochir (Wien). 2020; 162(7):1771-1775.

24. Kim AH, Tatter S, Rao G, et al. Laser Ablation of Abnormal Neurological Tissue Using Robotic NeuroBlate System (LAANTERN): 12-month outcomes and quality of life after brain tumor ablation. Neurosurgery. 2020;nyaa071.

25. Shao J, Radakovich NR, Grabowski M, et al. Lessons learned in using laser interstitial thermal therapy for treatment of brain tumors: a case series of 238 patients from a single institution. World Neurosurg. 2020;139:e345-e354.
26. Sawaya R, Hammoud M, Schoppa D, et al. Neurosurgical outcomes in a modern series of 400 craniotomies for treatment of parenchymal tumors. Neurosurgery. 1998;42(5): 1044-1056.

27. Everink IH, van Haastregt JC, van Hoof SJ, et al. Factors influencing home discharge after inpatient rehabilitation of older patients: a systematic review. BMC Geriatr. 2016;16:5.

28. Leuthardt EC, Voigt J, Kim AH, Sylvester P. A single-center cost analysis of treating primary and metastatic brain cancers with either brain laser interstitial thermal therapy (LITT) or craniotomy. Pharmacoeconom Open. 2017;1(1):53-63.

29. Bell M, Eriksson LI, Svensson T, et al. Days at home after surgery: an integrated and efficient outcome measure for clinical trials and quality assurance. EClinicalMedicine. 2019;11:18-26.

\section{Disclosures}

The authors report no conflict of interest concerning the materials or methods used in this study or the findings specified in this paper.

\section{Author Contributions}

Conception and design: both authors. Acquisition of data: Ginalis. Analysis and interpretation of data: both authors. Drafting the article: both authors. Critically revising the article: both authors. Reviewed submitted version of manuscript: both authors. Approved the final version of the manuscript on behalf of both authors: Danish. Statistical analysis: Ginalis. Study supervision: Danish.

\section{Supplemental Information Videos \\ Video Abstract. https://vimeo.com/453634154.}

\section{Correspondence}

Shabbar F. Danish: Rutgers Robert Wood Johnson Medical School, New Brunswick, NJ. shabbar.danish@rutgers.edu. 